\title{
Factors Influencing the Analytical Performance of an Atmospheric Sampling Glow Discharge Ionization Source as Revealed via Ionization Dynamics Modeling
}

\author{
Douglas E. Goeringer \\ Organic and Biological Mass Spectrometry Group, Chemical Sciences Division, Oak Ridge National Laboratory, \\ Oak Ridge, Tennessee, USA
}

\begin{abstract}
A kinetic model is developed for the dynamic events occurring within an atmospheric sampling glow discharge that affect its performance as an ion source for analytical mass spectrometry. The differential equations incorporate secondary electron generation and thermalization, reagent and analyte ion formation via electron capture and ion-molecule reactions, ion loss via recombination processes, diffusion, and ion-molecule reactions with matrix components, and the sampling and pumping parameters of the source. Because the ion source has a flow-through configuration, the number densities of selected species can be estimated by applying the steady-state assumption. However, understanding of its operation is aided by knowledge of the dynamic behavior, so numerical methods are applied to examine the time dependence of those species as well. As in other plasma ionization sources, the ionization efficiency is essentially determined by the ratio of the relevant ion formation and recombination rates. Although thermal electron and positive reagent ion number densities are comparable, the electron capture/ion-molecule reaction rate coefficient ratio is normally quite large and the ion-electron recombination rate coefficient is about an order of magnitude greater than that for ion-ion recombination. Consequently, the efficiency for negative analyte ion formation via electron capture is generally superior to that for positive analyte ion generation via ion-molecule reaction. However, the efficiency for positive analyte ion formation should be equal to or better than that for negative analyte ions when both ionization processes occur via ion-molecule reaction processes (with comparable rate coefficients), since the negative reagent ion density is considerably less than that for positive reagent ions. Furthermore, the particularly high number densities of thermal electrons and reagent ions leads to a large dynamic range of linear response for the source. Simulation results also suggest that analyte ion number densities might be enhanced by modification of the standard physical and operating parameters of the source. (J Am Soc Mass Spectrom 2003, 14, 1315-1326) () 2003 American Society for Mass Spectrometry
\end{abstract}

$\mathrm{D}$ irect detection of organic compounds at trace levels in ambient air continues to be an important application of mass spectrometry [1]. Examples include air pollution monitoring [2], detection of explosives [3], and characterization of emissions from the body [4]. In these and other applications, a number of methods have been utilized for generation of ions from compounds of interest. Electron ionization (EI) has been employed for formation of positive ions in situations for which air is leaked directly into the mass spectrometer $[5,6]$. To enhance ionization selectivity over that exhibited by EI, single- and multi-photon ionization techniques have been used for determination

Published online September 13, 2003

Address reprint requests to Dr. D. E. Goeringer, Organic and Biological Mass Spectrometry Group, Chemical Sciences Division, Oak Ridge National Laboratory, Bldg. 4500-S, MS 6131, Oak Ridge, TN 37831-6131, USA. E-mail: goeringerde@ornl.gov of targeted aromatic compounds in automobile exhaust [7-10]. More widely used approaches for direct sampling are based on atmospheric pressure chemical ionization (APCI) [11] which is capable of making positive or negative ions. APCI is effected by a sequence of electron- and ion-molecule reactions initiated by electrons that are generally produced via either a corona discharge [12] or a $\beta$-emitter such as ${ }^{63} \mathrm{Ni}[1,13]$. Because the equilibrium distribution in such reactions under normal APCI operating conditions often favors terminal ions that are characteristic of trace impurities rather than major components of the support gas [14], the ionization method has proven to be highly sensitive in analytical applications. For the same reason, however, APCI can be subject to matrix effects from compounds that compete for charge.

Our laboratory has developed another ionization technique, atmospheric sampling glow discharge ion- 
ization (ASGDI), for use in the analysis of trace amounts of organic vapors in ambient air [15]. The ASGDI source is simple and robust, exhibiting a rapid and memoryfree response to gas-phase samples. In addition, it has been demonstrated to be very sensitive for a variety of heteroatom-containing organic compounds. As with conventional APCI sources, ionization processes in the ASGDI source are initiated by electrons, and ionization occurs primarily via electron- and ion-molecule reactions. However, in the latter case electrons and reagent ions are produced in a reduced pressure glow discharge with ambient air serving as both the support gas and the source of reagent precursor compounds. Furthermore, the glow discharge is maintained in the shockwave structure created by the gas dynamics of the sample inlet free-jet expansion, so that ionization yields may be altered by manipulation of the mean molecular flow [16].

The effect of instrumental parameters on performance of the ASGDI source in analytical mass spectrometry have been delineated previously $[15,16]$. However, a rigorous kinetic analysis of the fundamental processes governing its operation has not been made. Ion sources can be characterized with regard to their dominant ion loss mechanism. Ions are lost primarily by drift due to electric fields or by diffusion when they are present at relatively low number densities such as exist in standard EI and chemical ionization (CI) sources. At the other extreme in ion number density are unipolar devices such as the corona APCI source [12, 17], for which ion loss due to drift in the electric field created by the space charge becomes dominant. In contrast to corona APCI, charged particles of both polarities are present in bipolar ion sources. There the ion and electron number densities are sufficiently high that diffusion becomes ambipolar, so that the dominant ion loss process then becomes recombination (ionelectron or ion-ion). Such recombination-dominated conditions exist in the high pressure electron capture (HPEC) [18-20], ${ }^{63} \mathrm{Ni}$ APCI, and ASGDI sources. The factors influencing performance of the $\mathrm{HPEC},{ }^{63} \mathrm{Ni}$ APCI, and corona APCI ion sources have been examined theoretically using detailed kinetic treatments [2123], the results enabling subsequent improvements in performance and applicability. To achieve similar advancements in the ASGDI source, progress in understanding the basis for its response is also desirable. This paper draws upon the insight provided by the above studies to develop a kinetic model for ionization dynamics in the atmospheric sampling glow discharge, and then uses it to determine the influence of fundamental ion processes and operating parameters on performance of the ASGDI source for analytical mass spectrometry.

\section{Results and Discussion}

\section{Basic Operation}

Source description. Because a detailed description of an ASGDI source as applied to the detection of trace

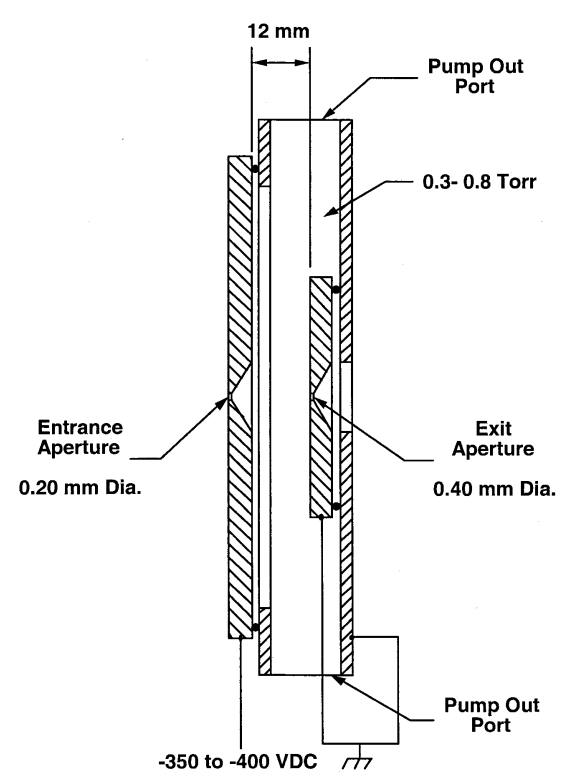

Figure 1. Diagram of the ASGDI source.

organics in ambient air has previously been presented [15], only the salient characteristics of the apparatus pertaining to its dynamics will be presented here. The standard configuration, shown schematically in Figure 1 , is comprised of a stainless steel, right cylinder $(\sim 4 \mathrm{~cm}$ diameter) with the ends enclosed by two electrically isolated, stainless steel, circular plates. The end plates, each having an aperture at the center, are spaced $\sim 12$ $\mathrm{mm}$ apart. Introduction of air and neutral analyte and matrix molecules occurs directly from the ambient atmosphere via the front aperture ( $\sim 200 \mu \mathrm{m}$ diameter $)$. The steady-state operating pressure $P_{\text {ASGDI }}(\sim 0.3-0.8$ torr) in the discharge region between the plates is maintained by continuous evacuation of the ion source chamber via four pumping ports on its periphery. The glow discharge is normally established by applying a potential ( -350 to $-400 \mathrm{VDC})$ to the sampling end plate with the cylinder and exit end plate both fixed at ground. Charged species of both polarities emerge from the exit aperture $(\sim 400 \mu \mathrm{m}$ diameter $)$ when this configuration is used.

Ion production processes. The major negatively charged species in the discharge are electrons. Initially formed in the cathode fall region with high energy due to the large potential drop occurring there, fast electrons subsequently ionize support gas molecules. Acceleration of the positive ions into the cathode can then cause ejection of additional electrons thereby sustaining the glow discharge. As in conventional atmospheric pressure ionization, a sequence of rapid ion-molecule reactions subsequent to the initial electron ionization event results in the formation of relatively stable hydronium ion-water clusters $\mathrm{H}_{3} \mathrm{O}^{+}\left(\mathrm{H}_{2} \mathrm{O}\right)_{\mathrm{n}}$ [24]. The other major positively charged ions are $\mathrm{O}_{2}^{+\cdot}$ and $\mathrm{NO}^{+}$. The secondary electrons generated in the initial ionization process are rapidly thermalized by collisions with the support 
gas as well. Electron capture of the thermal electrons then produces negatively charged ions such as $\mathrm{O}_{2}^{-}$, $\left(\mathrm{H}_{2} \mathrm{O}\right) \mathrm{O}_{2}^{-\cdot}$, and $\mathrm{NO}_{2}^{-}$.

A variety of analyte ion formation and conversion processes can occur in glow discharges [25], the complexity of which increases when the discharge is supported by a mixture of gases such as in the ASGDI source. Some general examples of such processes are depicted below.

$$
\begin{array}{cll}
\mathrm{A}+\mathrm{e}_{\text {therm }}^{-} \rightarrow \mathrm{A}^{-} & \text {A } & \\
\mathrm{A}+\mathrm{R}^{-} \stackrel{k_{i M}}{\rightarrow} \mathrm{A}^{-}+\mathrm{R} & \text { B } & \mathrm{A} \equiv \text { analyte } \\
\mathrm{AH}+\mathrm{R}^{-} \stackrel{k_{i M}}{\rightarrow} \mathrm{A}^{-}+\mathrm{RH} & \mathrm{C} & \begin{array}{l}
\mathrm{R} \equiv \text { reagent } \\
\mathrm{P}^{+} \equiv \text { positive ion }
\end{array} \\
\mathrm{A}+\mathrm{PH}^{+} \stackrel{k_{i M}}{\rightarrow} \mathrm{AH}^{+}+\mathrm{P} & \text { D } & \mathrm{e}_{\text {therm }}^{-} \equiv \text { thermal electron } \\
\mathrm{A}+\mathrm{P}^{+} \stackrel{k_{i M}}{\rightarrow} \mathrm{A}^{+}+\mathrm{P} & \text { E } &
\end{array}
$$

For compounds having a positive electron affinity, analyte anions can be formed via electron capture of thermal electrons (Reaction A); $k_{e C}$ will be used to represent the reaction rate coefficient for that process. Collisional thermalization of secondary electrons is a process of great importance to ASGDI sensitivity because the rate coefficients of electron capture reactions are often quite high at very low electron energies [26]. The electron thermalization rate must be appreciably greater than that for electron loss in order to maintain a relatively high steady-state number density of thermal electrons. In addition, the thermal electron capture rate must be sufficiently rapid to allow appreciable anion formation to occur during the available time for reaction. Charge exchange (Reaction $\mathrm{B}$ ) with negative reagent ions or proton abstraction (Reaction C) from acidic compounds by reagent anions can also yield analyte anions. Positively charged organic ions can be generated via proton transfer (Reaction D) from hydronium ion-water clusters to molecules with higher gasphase basicities than water. Organic cations are also formed via charge exchange reactions (Reaction $\mathrm{E}$ ) with the other major positive reagent ions $\mathrm{NO}^{+}$and $\mathrm{O}_{2}^{+\cdot}$. For Reactions B-E, $k_{i M}$ will be used to denote the ionmolecule reaction rate coefficient.

Signal generation. The current for any given ionic species entering the mass spectrometer through the ASGDI exit aperture is approximately equal to the product of its source number density near the aperture (at the point where ions become entrained in the flow through the aperture) and the conductance of the aperture. Since the conductance is independent of ionic species, relative ion currents are expected to be reflective of the relative magnitudes of ion number densities as determined by the kinetic model.

\section{Functional Dynamics}

The overall ASGDI dynamics were modeled by combining a system of coupled differential equations describing the rates of quiescent source processes and formation/loss for analyte species with the physical parameters of the source. The hydrodynamic properties of the analyte inlet free-jet expansion, although intrinsic to ASGDI processes, are outside the scope of this study, and thus, not included here. Although the populations of various species $X$ in the source are interdependent, their steady-state number densities can be estimated by applying the steady-state assumption to each (i.e., $\mathrm{d}[\mathrm{X}] / \mathrm{d} t=0)$ because the source has a flow-through configuration. However, understanding ASGDI performance is aided by knowledge of its dynamic behavior, so numerical methods are applied here to model and examine the time dependence of various species within the source as well. The following example calculations assume that the support gas is primarily air at pressure $P_{\text {ASGDI }}=0.8$ torr and temperature $T=300 \mathrm{~K}$, and that the analyte ion has a mass of $200 \mathrm{Da}$. Symbols used to represent the individual species, rate coefficients, and operational parameters for the main processes involved are compiled in Table 1.

Quiescent discharge conditions. Quiescent conditions refer here to the ASGDI source for which the ionization voltage is applied, but no analyte is present. The production of secondary electrons (and positive ions) via fast electron ionization in the quiescent source is offset by their loss due to recombination with positive ions (and electrons, negative ions), diffusion to the source walls, and ventilation. In addition, secondary electrons are thermalized by collisions with the support gas and subsequently may be captured by neutral species to form negatively charged reagent ions. Thus, although electrons are the dominant negative charge carriers in the ASGDI source, negative reagent ions can also be present at significant number densities. Negative ion loss occurs through recombination with positive ions in addition to removal via diffusion and ventilation. In addition to ionization, neutrals entering via the inlet aperture are depleted by ventilation and diffusion accompanied by any subsequent chemical reaction with the walls. Given the above, five coupled differential equations can be written for the rate of change in secondary electron number density $\left[e_{\text {sec }}^{-}\right]$, thermal electron number density $\left[e_{\text {therm }}^{-}\right]$(where $\left[e_{\text {total }}^{-}\right]=$ $\left.\left[e_{\text {sec }}^{-}\right]+\left[e_{\text {therm }}^{-}\right]\right)$, positive ion number density $\left[P^{+}\right]$, and reagent anion $\left[R^{-}\right]$and neutral $[R]$ number densities in the quiescent ASGDI source:

$$
\begin{aligned}
\frac{\mathrm{d}\left[e_{\mathrm{sec}}^{-}\right]}{\mathrm{d} t}= & S_{e P} / V-\left(k_{\text {therm }}+R_{P^{+}-e^{-}}\left[P^{+}\right]+D_{a} / \Lambda^{2}\right. \\
& +F / V)\left[e_{\mathrm{sec}}^{-}\right]
\end{aligned}
$$


Table 1. Symbols used for ion processes and operational parameters

\begin{tabular}{|c|c|c|}
\hline Symbol & Definition & Typical value \\
\hline$\left[A_{0}\right]$ & analyte molecule number density external to source & variable \\
\hline$[A]$ & analyte molecule number density inside the source & variable \\
\hline$\left[A^{-}\right]$ & analyte anion number density & variable \\
\hline$\left[A^{+}\right]$ & analyte cation number density & variable \\
\hline$\left[e_{s e c}^{-}\right]$ & secondary electron number density ${ }^{a}$ & $1.4 \times 10^{10} \mathrm{~cm}^{-3}$ \\
\hline$\left[e_{t h e r m}^{-s^{-1}}\right]$ & thermal electron number density ${ }^{\mathrm{a}}$ & $5.3 \times 10^{10} \mathrm{~cm}^{-3}$ \\
\hline$\left[P^{+}\right]^{\text {therm }}$ & positive ion number density ${ }^{a}$ & $6.7 \times 10^{10} \mathrm{~cm}^{-3}$ \\
\hline$\left[R^{-}\right]$ & reagent anion $\left(\mathrm{O}_{2}^{-}\right)$number density ${ }^{\mathrm{a}}$ & $4.0 \times 10^{8} \mathrm{~cm}^{-3}$ \\
\hline$[R]$ & reagent neutral $\left(\mathrm{O}_{2}\right)$ number density inside the source ${ }^{a}$ & $5 \times 10^{15} \mathrm{~cm}^{-3}$ \\
\hline$\left[M_{0}\right]$ & matrix neutral number density external to source & variable \\
\hline$[M]$ & matrix neutral number density inside the source & variable \\
\hline$[N]$ & support gas number density inside the source ${ }^{a}$ & $2.5 \times 10^{16} \mathrm{~cm}^{-3}$ \\
\hline$k_{\mathrm{eC}}$ & electron capture rate coefficient [37] & $1 \times 10^{-7} \mathrm{~cm}^{3}-\mathrm{s}^{-1}$ \\
\hline$k_{i M}$ & ion-molecule reaction rate coefficient [37] & $1 \times 10^{-9} \mathrm{~cm}^{3}-\mathrm{s}^{-1}$ \\
\hline$k_{\text {therm }}$ & electron thermalization rate coefficient at $P_{A S G D I}$ [28] & $2.4 \times 10^{5} \mathrm{~s}^{-1}$ \\
\hline$k_{e R}$ & rate coefficient of $\mathrm{O}_{2}^{-\cdot}$ formation at $P_{A S G D I}{ }^{a}$ & $7.2 \times 10^{-14} \mathrm{~cm}^{3}-\mathrm{s}^{-1}$ \\
\hline$S_{e P}$ & ion-electron pair formation rate ${ }^{a}$ & $6.2 \times 10^{16} \mathrm{~s}^{-1}$ \\
\hline$R_{P}^{+}-e^{-}$ & positive ion-electron recombination coefficient [34] & $9 \times 10^{-7} \mathrm{~cm}^{3}-\mathrm{s}^{-1}$ \\
\hline$R_{\text {pos-neg }}$ & ion-ion recombination coefficient [35] & $7 \times 10^{-8} \mathrm{~cm}^{3}-\mathrm{s}^{-1}$ \\
\hline$D_{a}$ & ambipolar diffusion coefficient ${ }^{\mathrm{a}}$ & $1.2 \times 10^{2} \mathrm{~cm}^{2}-\mathrm{s}^{-1}$ \\
\hline$D_{n}$ & neutral diffusion coefficient ${ }^{a}$ & $5.1 \times 10^{1} \mathrm{~cm}^{2}-\mathrm{s}^{-1}$ \\
\hline$\Lambda$ & characteristic diffusion length [32] & $0.3 \mathrm{~cm}$ \\
\hline SC & inlet aperture conductance & $5 \mathrm{~cm}^{3}-\mathrm{s}^{-1}$ \\
\hline$F$ & flow rate through source ${ }^{a}$ & 5 liter-s ${ }^{-1}$ \\
\hline$V$ & source volume & $15 \mathrm{~cm}^{3}$ \\
\hline$P_{A S G D I}$ & source operating pressure & 0.8 Torr \\
\hline$P_{\text {sample }}$ & sample pressure external to source & Ambient \\
\hline
\end{tabular}

aParameter value from calculation (see text).

$$
\begin{aligned}
& \frac{\mathrm{d}\left[e_{\text {therm }}^{-}\right]}{\mathrm{d} t}=k_{\text {therm }}\left[e_{\mathrm{sec}}^{-}\right]-\left(k_{e R}[R]+R_{P^{+}-e^{-}}\left[P^{+}\right]\right. \\
& \left.+D_{a} / \Lambda^{2}+F / V\right)\left[e_{\text {therm }}^{-}\right] \\
& \frac{\mathrm{d}\left[P^{+}\right]}{\mathrm{d} t}=S_{e P} / V-\left(R_{P^{+-} e^{-}}\left[e_{\text {total }}^{-}\right]+R_{\text {pos-neg }}\left[R^{-}\right]\right. \\
& \left.+D_{a} / \Lambda^{2}+F / V\right)\left[P^{+}\right] \\
& \frac{\mathrm{d}\left[R^{-}\right]}{\mathrm{d} t}=k_{e R}\left[e_{\text {therm }}^{-}\right][R]-\left(R_{\text {pos-neg }}\left[P^{+}\right]+D_{a} / \Lambda^{2}\right. \\
& +F / V)\left[R^{-}\right] \\
& \frac{\mathrm{d}[R]}{\mathrm{d} t}=(S C / V)\left[R_{0}\right]-\left(k_{e R}\left[e_{\text {therm }}^{-}\right]+D_{n} / \Lambda^{2}+F / V\right) \\
& \times[R]
\end{aligned}
$$

Values of parameters for the various fundamental processes occurring in the ASGDI source are discussed below; the simulations following assume the same values unless otherwise noted. Because one secondary electron-ion pair $\left(e^{-}-P^{+}\right)$is formed per about $35 \mathrm{eV}$ of energy in each fast electron [27], the total ionization rate $S_{e P}$ of support gas neutrals is

$$
S_{e P}=\frac{V_{i o n i z} \cdot i_{i o n i z}}{35 e V}
$$

where $i_{\text {ioniz }}$ is the ionizing electron current and $V_{\text {ioniz }}$ is its energy. Operating conditions of $V_{\text {ioniz }}=-350$ volt and $i_{\text {ioniz }}=1 \mathrm{~mA}$ yield $S_{e P}=6.2 \times 10^{16} \mathrm{~s}^{-1}$. The electron thermalization rate coefficient $k_{\text {therm }}$ is $3.0 \times 10^{5} \mathrm{~s}^{-1}$ in 1 torr of $\mathrm{N}_{2}$ [28], and it is directly related to the $\mathrm{N}_{2}$ number density over at least a range of $0.1-1$ torr. $\mathrm{O}_{2}$ is assumed to be the major anion-forming neutral reagent. The third order rate constants $k_{e R}$ for reactions

$$
\mathrm{O}_{2}+\mathrm{e}^{-}+\mathrm{O}_{2} \rightarrow \mathrm{O}_{2}^{-\cdot}+\mathrm{O}_{2}
$$

and

$$
\mathrm{O}_{2}+\mathrm{e}^{-}+\mathrm{N}_{2} \rightarrow \mathrm{O}_{2}^{-\cdot}+\mathrm{N}_{2}
$$

are $1.4 \times 10^{-29} \mathrm{~cm}^{6}-\mathrm{s}^{-1}$ and $1.1 \times 10^{-31} \mathrm{~cm}^{6}-\mathrm{s}^{-1}$, respectively $[29,30]$. Assuming $P_{\text {ASGDI }} \approx 0.8$ torr, then $\left[\mathrm{O}_{2}\right] \approx 5.2 \times 10^{15} \mathrm{~cm}^{-3}$ and $\left[\mathrm{N}_{2}\right] \approx 2.1 \times 10^{16} \mathrm{~cm}^{-3}$, and $k_{e R}$ can be taken as second order with a value of $\sim 7.3 \times$ $10^{-14} \mathrm{~cm}^{3}-\mathrm{s}^{-1}$.

The flow rate $F$ through the source for our ASGDI configuration can be approximated from the conductance of the front aperture $S C\left(\sim 5 \mathrm{~cm}^{3}-\mathrm{s}^{-1}\right)$ and the 
ambient sample pressure $P_{\text {sample }}(\sim 760$ torr) according to

$$
F=\frac{S C \cdot P_{\text {sample }}}{P_{\text {ASGDI }}}
$$

which yields $F \approx 4.751-\mathrm{s}^{-1}$ [31]. For our estimated source volume $V\left(\sim 15 \mathrm{~cm}^{3}\right)$, the rate coefficient for sampling $S C / V$ is $\sim 3.3 \times 10^{-1} \mathrm{~s}^{-1}$ and for ventilation $F / V$ is $\sim 3.2 \times 10^{2} \mathrm{~s}^{-1}$. The magnitude of the neutral diffusion coefficient $D_{n}$ is given by the expression

$$
D_{n}=\frac{3}{8} \frac{\sqrt{\frac{\pi k T}{2 \mu_{A-\text { neut }}}}}{\pi d_{A-\text { neut }}^{2}\left(\frac{P_{A S G D I}}{T}\right) L_{c}}
$$

where $\mu_{A-n e u t}$ is the analyte-neutral reduced mass, $d_{A-\text { neut }}$ is their average molecular diameter, and $L_{c}$ is the Loschmidt constant. Assuming that $d_{A-n e u t} \approx 6 \times 10^{-8}$ $\mathrm{cm}$ yields $D_{n} \approx 51 \mathrm{~cm}^{2}-\mathrm{s}^{-1}$. Using the method of McDaniel [32] to determine $\Lambda$ for a right cylinder, $\Lambda$ is estimated to be $\sim 0.30 \mathrm{~cm}$ for our ASGDI configuration. The rate coefficient for neutral diffusion $D_{n} / \Lambda^{2}$ is then $\sim 5.6 \times 10^{2} \mathrm{~s}^{-1}$.

As will become evident in the calculations below, the ion-electron number densities in the ASGDI source are sufficiently large that the Debye length $(\sim 1 \mu \mathrm{m})$ is small compared to the source dimensions. Thus, although the free diffusion coefficient for electrons $\left(D_{e}\right)$ is approximately three orders of magnitude larger than for positive ions $\left(D_{+}\right)$, independent transport of the species cannot occur. Coupling due to electron-ion interaction causes the ion diffusion rate to be somewhat higher and the electron diffusion rate to be significantly lower. The result is that a single (ambipolar) diffusion coefficient $\left(D_{a}\right)$ applies to both electrons and positive ions [32]. Furthermore, $D_{a}$ also applies to negative ions because their diffusion is coupled to positive ion diffusion. $D_{a}$ can be evaluated from the free positive ion diffusion coefficient $\left(D_{a}=2 D_{+}\right)$, which in turn can be obtained from the Einstein equation,

$$
D_{+}=\frac{K_{p o l} k T}{e}
$$

where the polarization limit of ion mobility $K_{p o l}$ is [33]

$$
\begin{aligned}
K_{\text {pol }}= & \frac{13.853}{\sqrt{\left(\frac{\alpha_{\text {neut }}}{\AA^{3}}\right)\left(\frac{\mu_{\text {ion-neut }}}{\text { Dalton }}\right)}}\left(\frac{\frac{T}{K}}{273.15}\right) \\
& \times\left(\frac{760}{\frac{P_{\text {ASGDI }}}{\text { Torr }}}\right) \frac{\mathrm{cm}^{2}}{\text { volt-sec }}
\end{aligned}
$$

$\mu_{\text {ion-neut }}$ is the ion-neutral reduced mass in Daltons, and $\alpha_{\text {neut }}$ is the polarizability of the neutral in $\AA^{3}$. Using typical ASGDI operating conditions $\left(\alpha_{\text {neut }}=1.74 \AA^{3}\right.$ for $\mathrm{N}_{2}$ ) [33], $D_{a}$ is calculated to be $\sim 115 \mathrm{~cm}^{2}-\mathrm{s}^{-1}$. Substituting the estimate given above for $\Lambda$ then yields a value of $\sim 1.3 \times 10^{3} \mathrm{~s}^{-1}$ for the ambipolar diffusion rate coefficient $D_{a} / \Lambda^{2}$.

Under normal ASGDI operating conditions ion-electron dissociative recombination is a two-body process, so the recombination coefficient $R_{\mathrm{p}-e^{-}}^{+}$is independent of pressure. Furthermore, as the complexity of the positive ion increases, the recombination coefficient approaches a constant value that can be estimated by [34].

$$
R_{P^{+}-e^{-}}=9 \times 10^{-7} \mathrm{~cm}^{3} \mathrm{~s}^{-1} \sqrt{\frac{300 \mathrm{~K}}{T}}
$$

The ion-ion recombination coefficient $R_{\text {pos-neg }}$ for polyatomic ions is essentially independent of ion size, but the coefficient can be pressure dependent since a competition exists between two- and three-body processes. However, the two-body process is dominant at pressures below $\sim 1$ torr [35], so $R_{\text {pos-neg }}$ has virtually no pressure dependence in the pressure regime of interest in the ASGDI source. Thus, for any positive ion-negative ion pair, a good estimate of $R_{\text {pos-neg }}$ is provided by that for $\mathrm{NO}^{+}$and $\mathrm{NO}_{2}^{-}$as given by [35]:

$$
R_{\text {pos-neg }}=6.8 \times 10^{-7} \mathrm{~cm}^{3} \mathrm{~s}^{-1}\left(T^{-0.4}\right)
$$

Thus, $R_{\text {pos-neg }}=6.9 \times 10^{-8} \mathrm{~cm}^{3}-\mathrm{s}^{-1}$ is approximately an order of magnitude less than $R_{\mathrm{P}-e^{-}}^{+}$at $300 \mathrm{~K}$. This is in contrast to atmospheric pressure weak plasma ion sources in which ion-ion recombination is governed by a three-body process. In such cases, a single ion-electron and ion-ion recombination coefficient approximately equal to $1 \times 10^{-6} \mathrm{~cm}^{3}-\mathrm{s}^{-1}$ is assumed [14].

To determine the time-dependent behavior of charged species in the quiescent source, simulation conditions were adjusted so that the support gas neutral population had reached a steady-state concentration prior to the discharge initiation (at $t=2.5 \mathrm{~ms}$ ). The coupled system of differential eqs $1-5$ was then solved to obtain numerical estimates for the time-dependent number densities of each charged species. Because $D_{a} / \Lambda^{2} \gg F / V$, the concentration of such species should be relatively uniform throughout the ASGDI source except for the free-jet expansion core. Figure 2 shows that overall charge balance is maintained as the electrons and positive ions reach their steady-state concentrations $\left(\left[e_{\mathrm{sec}}^{-}\right] \approx 1.4 \times 10^{10} \mathrm{~cm}^{-3},\left[e_{\text {therm }}^{-}\right] \approx 5.3 \times 10^{10}\right.$ $\left.\mathrm{cm}^{-3},\left[P^{+}\right] \approx 6.7 \times 10^{10} \mathrm{~cm}^{-3}\right)$ quite rapidly $(<40 \mu \mathrm{s})$. The reagent anion number density $\left(\left[R^{-}\right] \approx 4.0 \times 10^{8}\right.$ $\mathrm{cm}^{-3}$ ) requires a somewhat longer time. This disparity stems from the delay in buildup of the thermal electron population coupled with the relatively small $k_{e C}$ associated with electron capture by $\mathrm{O}_{2}$. The results indicate 


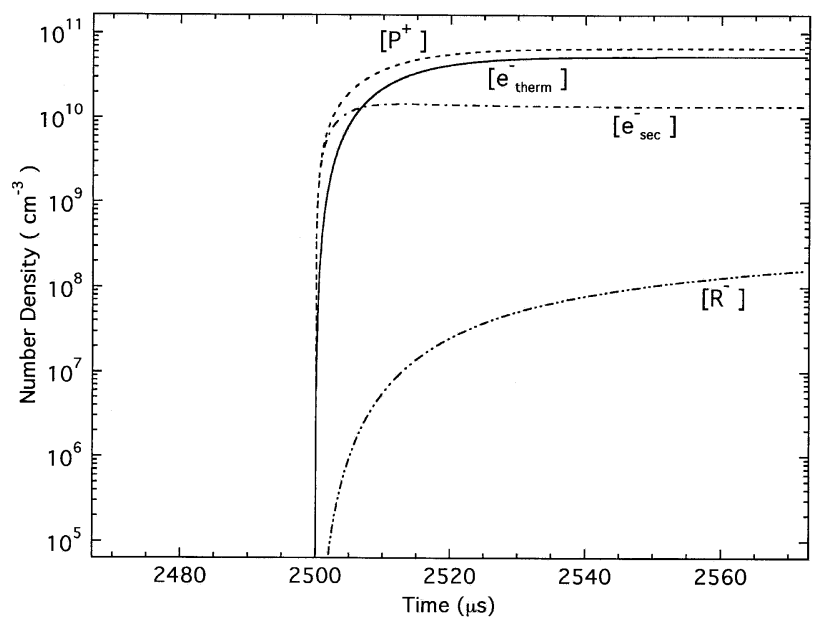

Figure 2. Time dependence of selected species in quiescent ASGDI source following discharge initiation for electron-ion pair generation rate $S_{e P}=6.2 \times 10^{16} \mathrm{~s}^{-1}$.

that the thermal electron generation rate $\left(\sim 7.5 \times 10^{14}\right.$ $\left.\mathrm{e}^{-}-\mathrm{s}^{-1}\right)$ is sufficiently greater than the overall electron loss rate to yield $\left[e_{\text {therm }}^{-}\right] /\left[e_{\text {total }}^{-}\right] \approx 0.8$ at steady-state. In addition, the positive ion number density is seen to be substantially larger than the negative ion number density, an effect observed experimentally in the ASGDI source [15]. The calculated $\left[P^{+}\right]$values are also consistent with those predicted and experimentally observed [21] in the HPEC source, which is also recombinationdominated. Figure 3 shows the dependence of $\left[e_{\mathrm{sec}}^{-}\right]$, $\left[e_{\text {therm }}^{-}\right],\left[P^{+}\right]$, and $\left[R^{-}\right]$at steady-state on $S_{e P}$ from $\sim 10^{14}-10^{17} \mathrm{~s}^{-1}$. Overall charge balance is maintained as electron and positive ion number densities increase with $S_{e P}$. However, $\left[e_{\text {therm }}^{-}\right] /\left[P^{+}\right]$and $\left[R^{-}\right] /\left[P^{+}\right]$are observed to decrease as the $e_{\text {sec }}^{-} P^{+}$and $R^{-}-P^{+}$recombination rates become increasing competitive with those for $e_{\mathrm{sec}}^{-}$thermalization and formation of $R^{-}$via electron capture, respectively.

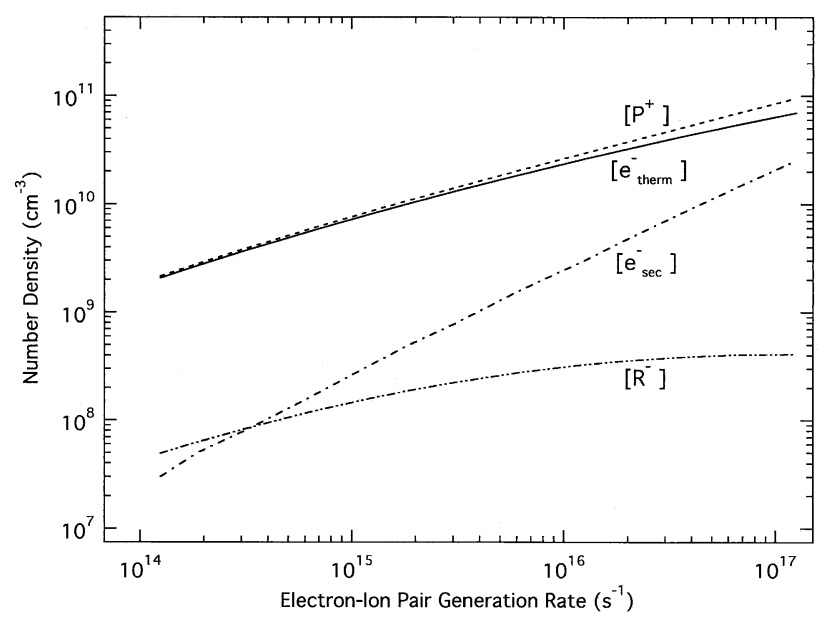

Figure 3. Relative steady-state concentrations of selected species in quiescent ASGDI source as a function of electron-ion pair production rate $S_{e P}$.
Overall rate equations. As indicated above, analyte anions in the ASGDI model are assumed to be formed via thermal electron capture and ion/molecule reactions (e.g., charge exchange, proton abstraction), and positive analyte ions are presumed to be generated via ion/ molecule reactions (e.g., charge exchange, proton transfer). Because electron capture rate coefficients can be quite large, the maximum theoretical value at room temperature being $\sim 5 \times 10^{-7} \mathrm{~cm}^{3}-\mathrm{s}^{-1}$ [36], $k_{e C}$ for analyte anion formation was taken to be $10^{-7} \mathrm{~cm}^{3}-\mathrm{s}^{-1}$. The rate coefficients for fast ion/molecule reactions are some two orders of magnitude lower [37], therefore $k_{i M}$ was assumed to be $10^{-9} \mathrm{~cm}^{3}-\mathrm{s}^{-1}$. It is appealing to describe the ASGDI ionization dynamics by methods similar to those used to explain the relative sensitivities in corona atmospheric pressure ionization mass spectrometry (APCI-MS); the rate expression for analyte ion intensities (under kinetic control) in the corona APCI source is [38]:

$$
\frac{d\left[A^{+}\right]}{d t}=k_{i M}\left[P^{+}\right][A]
$$

$[A]$ and $\left[A^{+}\right]$are the number densities for the analyte neutrals and ions, respectively. Since the concentration of the reagent species $\left[\mathrm{P}^{+}\right]$is significantly greater than that for analyte molecules at trace (ppb) levels, the expected time dependent behavior for analyte ions is then:

$$
\left[A^{+}\right]=[A]\left(1-\exp \left\{-k_{\text {ioniz }} \tau_{A}\right\}\right)
$$

where $k_{\text {ioniz }}$ is a pseudo first-order rate constant given by $k_{\text {ioniz }}=k_{i M}\left[P^{+}\right]$, and $\tau_{A}$ is the analyte reaction time (i.e., its drift time in the source). Because ions of only one polarity are present in the corona APCI source, essentially no analyte ion recombination loss occurs in the source drift space between the ionization region and the mass spectrometer inlet orifice. Instead, the corona APCI source is space-charge dominated. Thus, additional theoretical models have been developed that explain the variation in absolute sensitivities as a consequence of ion drift to the source walls [23].

In contrast to the corona APCI source, essentially equal concentrations for charged particles of both polarities are present in the ASGDI source. Such conditions preclude space-charge-induced electric fields. Thus, the dominant ion loss mechanism is ion-ion or ion-electron recombination. Consequently, analyte ion number densities are not described by the simple pseudo first-order kinetic expressions above. Therefore, the rate eqs 1-5 for quiescent ASGDI operation have been modified to form the overall ASGDI rate equations by including analyte ion loss due to recombination, in addition to analyte ion and neutral removal via diffusion and pumping. Matrix species $M$ that are entrained with the sample can affect ASGDI performance as well, so the overall rate equations also include terms for 
elimination of analyte ions via ion-molecule reactions with $M$ neutrals. In addition, the overall equations below have been simplified by assuming that number densities for reagent ions always greatly exceed those for analyte ions (i.e., $\left[R^{-}\right],\left[P^{+}\right] \gg\left[A^{-}\right],\left[A^{+}\right]$). Therefore, the latter are not included in the recombination terms. Finally, eq 14 is identical to eq 5 but is included again here for convenience.

$$
\begin{aligned}
\frac{\mathrm{d}[\mathrm{A}]}{\mathrm{d} t}= & (S C / V)\left[A_{0}\right]-\left(k_{e C}\left[e_{\text {therm }}^{-}\right]+k_{i M}\left\{\left[R^{-}\right]\right.\right. \\
& \left.\left.+\left[P^{+}\right]\right\}+D_{n} / \Lambda^{2}+F / V\right)[A] \\
\frac{\mathrm{d}[M]}{\mathrm{d} t}= & (S C / V)\left[M_{0}\right]-\left(k_{i M}\left\{\left[A^{-}\right]+\left[A^{+}\right]\right\}\right. \\
& \left.+D_{n} / \Lambda^{2}+F / V\right)[M]
\end{aligned}
$$

$$
\begin{aligned}
\frac{\mathrm{d}\left[\mathrm{A}^{-}\right]}{\mathrm{d} t}= & \left(k_{e C}\left[e_{\text {therm }}^{-}\right]+k_{i M}\left[R^{-}\right]\right)[A]-\left(R_{\text {pos-neg }}\left[P^{+}\right]\right. \\
& \left.+k_{i M}[M]+D_{a} / \Lambda^{2}+F / V\right)\left[A^{-}\right]
\end{aligned}
$$

$$
\begin{aligned}
\frac{\mathrm{d}\left[\mathrm{A}^{+}\right]}{\mathrm{d} t}= & k_{i M}\left[P^{+}\right][A]-\left(R_{P^{+}-e^{-}}\left[e_{\text {total }}^{-}\right]+R_{\text {pos-neg }}\left[R^{-}\right]\right. \\
& \left.+k_{i M}[M]+D_{a} / \Lambda^{2}+F / V\right)\left[A^{+}\right]
\end{aligned}
$$

$$
\begin{aligned}
\frac{\mathrm{d}\left[e_{\mathrm{sec}}^{-}\right]}{\mathrm{d} t}= & S_{e P} / V-\left(k_{\text {therm }}+R_{P^{+-} e^{-}}\left[P^{+}\right]+D_{a} / \Lambda^{2}\right. \\
& +F / V)\left[e_{\mathrm{sec}}^{-}\right]
\end{aligned}
$$

$$
\begin{aligned}
\frac{\mathrm{d}\left[e_{\text {therm }}^{-}\right]}{\mathrm{d} t}= & k_{\text {therm }}\left[e_{\mathrm{sec}}^{-}\right]-\left(k_{e C}[A]+k_{e R}[R]\right. \\
& \left.+R_{P^{+}-e^{-}}\left[P^{+}\right]+D_{a} / \Lambda^{2}+F / V\right)\left[e_{\text {therm }}^{-}\right]
\end{aligned}
$$

$$
\begin{aligned}
\frac{\mathrm{d}\left[\mathrm{P}^{+}\right]}{\mathrm{d} t}= & S_{e P} / V-\left(k_{i M}[A]+R_{P^{+-} e^{-}}\left[e_{\text {total }}^{-}\right]\right. \\
& \left.+R_{\text {pos-neg }}\left[R^{-}\right]+D_{a} / \Lambda^{2}+F / V\right)\left[P^{+}\right]
\end{aligned}
$$

$$
\begin{aligned}
\frac{\mathrm{d}\left[\mathrm{R}^{-}\right]}{\mathrm{d} t}= & k_{e R}\left[e_{\text {therm }}^{-}\right][R]-\left(k_{i M}[A]+R_{\text {pos-neg }}\left[P^{+}\right]\right. \\
& \left.+D_{a} / \Lambda^{2}+F / V\right)\left[R^{-}\right]
\end{aligned}
$$

$$
\begin{aligned}
\frac{\mathrm{d}[R]}{\mathrm{d} t}= & (S C / V)\left[R_{0}\right]-\left(k_{e R}\left[e_{\text {therm }}^{-}\right]+D_{n} / \Lambda^{2}+F / V\right) \\
& \times[R]
\end{aligned}
$$

Incorporating the requisite parameters and charged

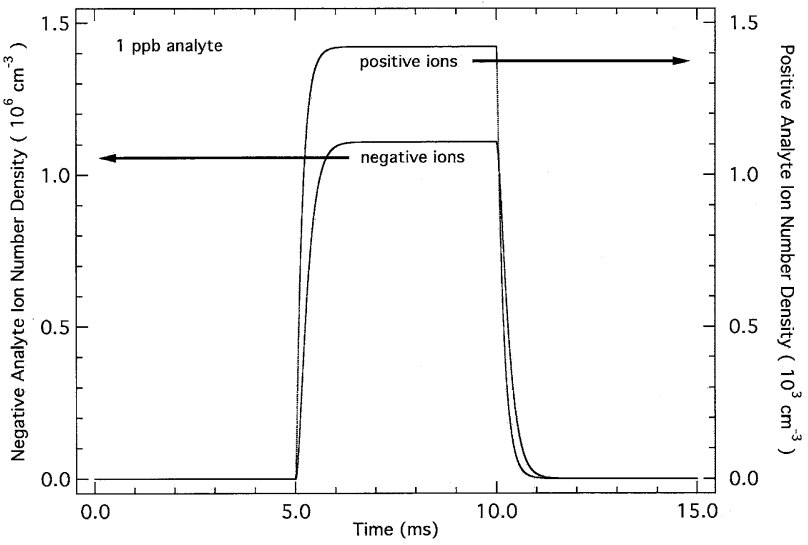

Figure 4. Time dependence of analyte ions in the ASGDI source corresponding to the introduction of a $5 \mathrm{~ms}$ pulse of analyte neutrals present at $1 \mathrm{ppb}$. Negative ions are produced via electron capture with $k_{e C}=10^{-7} \mathrm{~cm}^{3}-\mathrm{s}^{-1}$, positive ions via ion-molecule reaction with $k_{i M}=10^{-9} \mathrm{~cm}^{3}-\mathrm{s}^{-1}$.

particle number densities determined above, the analyte ion lifetime against subsequent loss via recombination, diffusion, or pumping, is then $\left(R_{\text {pos-neg }}\left[P^{+}\right]+\right.$ $\left.D_{a} / \Lambda^{2}+F / V\right)^{-1} \approx 1.6 \times 10^{-4} \mathrm{~s}$ for negative ions and $\left(R_{\mathrm{P}-e^{-}}^{+}\left[e_{\text {total }}^{-}\right]+D_{a} / \Lambda^{2}+F / V\right)^{-1} \approx 1.6 \times 10^{-5} \mathrm{~s}$ for positive ions. The lifetime for analyte molecules against ionization by electron capture or ion-molecule reaction is $\sim 1.5 \times 10^{-4} \mathrm{~s}$. Comparing the lifetimes against neutral ionization and ion loss with the lifetime against neutral loss by diffusion or pumping, $\sim 1.1 \times 10^{-3} \mathrm{~s}$, it is evident that a high probability exists for any particular neutral to be ionized and subsequently lost during its residence time.

Dynamic ion population and ionization efficiency. As in the previous calculations for the quiescent ASGDI source, numerical methods were used to obtain the time-dependent number densities of each species from the coupled overall rate eqs $6-14$. Figure 4 shows the simulated time-dependent behavior of the number densities for the analyte species in the ASGDI source with $\left[A_{0}\right]=1 \mathrm{ppb}$. In the simulations, the quiescent population of charged species was allowed to reach steadystate prior to admitting analyte in a $5 \mathrm{~ms}$ pulse. For situations in which negative ion formation occurs via electron capture, the number density of negative analyte ions is seen to be $\sim 780$ times larger than that for positive analyte ions (note that full-scale for $\left[A^{-}\right]$is $10^{3}$ times larger than for $\left.\left[A^{+}\right]\right)$. On the other hand, when the mechanism for negative ion formation is ion-molecule reaction, the number density of negative analyte ions is observed to be $\sim 15$ times smaller than that for positive analyte ions (not shown).

The principal parameters governing the analyte negative and positive ion number densities can be ascertained by simplifying eq 8 (using the assumption $R_{\text {pos-neg }}\left[P^{+}\right] \gg D_{a} / \Lambda^{2}, F / V$ ), and eq 9 (using the assumption $\left.R_{\mathrm{P}-e^{-}}^{+}\left[e_{\text {total }}^{-}\right] \gg R_{\text {pos-neg }}\left[R^{-}\right], D_{a} / \Lambda^{2}, F / V\right)$ to give the steady-state limits in eqs $15-17$. Eq 16 assumes that 
negative analyte formation via electron capture is not operative (i.e., $k_{e C}=0$ ). The effects of matrix components are ignored at this point (i.e., $\left[M_{0}\right],[M]=0$ ), but will be examined below.

$$
\left(\frac{\left[A^{-}\right]}{[A]}\right)_{e C} \approx \frac{k_{e C}\left[e_{\text {therm }}^{-}\right]}{R_{\text {pos-neg }}\left[P^{+}\right]}
$$

electron capture negative analyte ion formation

$$
\left(\frac{\left[A^{-}\right]}{[A]}\right)_{i M} \approx \frac{k_{i M}\left[R^{-}\right]}{R_{\text {pos-neg }}\left[P^{+}\right]}
$$

ion-molecule reaction negative analyte ion formation

$$
\frac{\left[A^{+}\right]}{[A]} \approx \frac{k_{i M}\left[P^{+}\right]}{R_{P^{+}-e^{-}\left[e_{\text {total }}^{-}\right]}}
$$

ion-molecule reaction positive analyte ion formation

Therefore, $\left[A^{-}\right] /[A]$ and $\left[A^{+}\right] /[A]$ are effectively determined by the ratio of their relevant ion formation and recombination rates (unless $F / V$ is greatly increased, or $\Lambda$ is significantly reduced, or both). In addition, the relative negative and positive analyte ion number densities can be estimated by dividing eqs 15,16 by eq 17 and using the approximation $\left[e_{\text {therm }}^{-}\right] \approx\left[e_{\text {total }}^{-}\right] \approx\left[P^{+}\right]$to give:

$$
\left(\frac{\left[A^{-}\right]}{\left[A^{+}\right]}\right)_{e C}=\frac{R_{P^{+}-e^{-}}}{R_{\text {pos-neg }}} \frac{k_{e C}}{k_{i M}}
$$

electron capture negative analyte ion formation

$$
\left(\frac{\left[A^{-}\right]}{\left[A^{+}\right]}\right)_{i M}=\frac{R_{P^{+-}-e^{-}}}{R_{\text {pos-neg }}} \frac{\left[R^{-}\right]}{\left[P^{+}\right]}
$$

ion-molecule reaction negative analyte ion formation

Thus, assuming that $R_{\mathrm{P}-e^{-}}^{+} / R_{\text {pos-neg }} \approx 10$ (vide supra), the ASGDI source would be expected to have $\left[A^{-}\right] /$ $\left[A^{+}\right] \approx 10^{3}$ (i.e., 1000 times better sensitivity for negative than positive analyte ions) when electron capture $\left(k_{e C}\right.$ $k_{i M} \approx 10^{2}$ ) is the dominant process for generating analyte anions. These predictions are corroborated by experimental results for 2,4,6-trinitrotoluene (TNT) [39], which presumably forms anions via electron capture due to its high electron affinity. In that work, positive ion detection limits for TNT were approximately three orders of magnitude higher than those for negative ions. Conversely, for situations in which the electron capture process is not operable, it is predicted that $\left[A^{-}\right] /\left[A^{+}\right] \leq$ 1 because electrons are the major negatively charged species in the discharge $\left(\left[R^{-}\right] \ll\left[e_{\text {total }}^{-}\right] \approx\left[P^{+}\right]\right)$.
The overall source efficiency $\left[A^{-}\right] /\left[A_{0}\right]$ and $\left[A^{+}\right] /$ $\left[A_{0}\right]$ (i.e., the fraction of analyte molecules in front of the inlet aperture that are present as analyte ions in the source and not lost via recombination, diffusion, or ventilation subsequent to ionization) can also be estimated. Simplifying eq 6 using the assumptions $k_{e C}\left[e_{\text {therm }}^{-}\right] \gg D_{n} / \Lambda^{2}, F / V \gg k_{i M}\left[P^{+}\right] \gg k_{i M}\left[R^{-}\right]$gives the steady-state expression for $[A] /\left[A_{0}\right]$, under electron capture dominated negative analyte ion formation conditions, in eq 20.

$$
\left(\frac{[A]}{\left[A_{0}\right]}\right)_{e C} \approx \frac{\frac{S C}{V}}{k_{e C}\left[e_{\text {therm }}^{-}\right]}=6.2 \times 10^{-5}
$$

Multiplying eq 20 by eqs 15 and 17 then gives eqs 21 and 22 representing the overall efficiencies for negative and positive analyte ion formation, respectively, under electron capture dominated conditions:

$$
\begin{aligned}
& \left(\frac{\left[A^{-}\right]}{\left[A_{0}\right]}\right)=\left(\frac{\left[A^{-}\right]}{[A]}\right)_{e C}\left(\frac{[A]}{\left[A_{0}\right]}\right)_{e C} \approx 7.2 \times 10^{-5} \\
& \left(\frac{\left[A^{+}\right]}{\left[A_{0}\right]}\right)=\frac{\left[A^{+}\right]}{[A]}\left(\frac{[A]}{\left[A_{0}\right]}\right)_{e C} \approx 7.0 \times 10^{-8}
\end{aligned}
$$

Similarly, the steady-state expression for $[A] /\left[A_{0}\right]$, under ion-molecule reaction dominated negative analyte ion formation conditions, from simplification of eq 6 is:

$$
\left(\frac{[A]}{\left[A_{0}\right]}\right)_{i M} \approx \frac{\frac{S C}{V}}{\frac{D_{n}}{\Lambda^{2}}+\frac{F}{V}}=3.8 \times 10^{-4}
$$

Eqs 24 and 25 then express the overall efficiencies for negative and positive analyte ion formation, respectively, under ion-molecule reaction dominated conditions:

$$
\begin{aligned}
& \left(\frac{\left[A^{-}\right]}{\left[A_{0}\right]}\right)_{i M}=\left(\frac{\left[A^{-}\right]}{[A]}\right)_{i M}\left(\frac{[A]}{\left[A_{0}\right]}\right)_{i M} \approx 3.3 \times 10^{-8} \\
& \left(\frac{\left[A^{+}\right]}{\left[A_{0}\right]}\right)_{i M}=\frac{\left[A^{+}\right]}{[A]}\left(\frac{[A]}{\left[A_{0}\right]}\right)_{i M} \approx 4.2 \times 10^{-7}
\end{aligned}
$$

Dividing eqs 21, 22, 24, and 25 by the sample dilution factor $\left(P_{\text {ASGDI }} / P_{\text {sample }} \approx 0.0011\right)$ of the ASGDI source gives the estimates shown in Table 2 for ionization efficiencies (i.e., the fraction of analyte molecules entering the source that are ionized and not lost subsequently via recombination, diffusion, or ventilation). The higher efficiency for positive ion formation when negative ions are generated via ion-molecule reaction rather than by electron capture, viz. $3.8 \times 10^{-4}$ versus $6.4 \times 10^{-5}$, simply reflects the increased availability of 
Table 2. Estimated ionization efficiencies

\begin{tabular}{|c|c|c|c|}
\hline & & $A^{-}$ & $A^{+}$ \\
\hline Analyte ion formation process & $\begin{array}{l}A^{-}: \text {electron capture }{ }^{\mathrm{b}} ; A^{+}: \text {ion-molecule reaction }{ }^{\mathrm{a}} \\
A^{-}, A^{+}: \text {ion-molecule reaction }\end{array}$ & $\begin{array}{l}6.5 \times 10^{-2} \\
3.0 \times 10^{-5}\end{array}$ & $\begin{array}{l}6.4 \times 10^{-5} \\
3.8 \times 10^{-4}\end{array}$ \\
\hline
\end{tabular}

aion-molecule reaction rate coefficient $k_{i M}=10^{-9} \mathrm{~cm}^{3}-\mathrm{s}^{-1}$; belectron capture rate coefficient $k_{e C}=10^{-7} \mathrm{~cm}^{3}-\mathrm{s}^{-1}$

neutral analyte due to the significantly lower negative ionization efficiency, viz. $3.0 \times 10^{-5}$ versus $6.5 \times 10^{-2}$.

Operating parameters. Because the overall rate equations include terms for various operating parameters, it is possible to simulate the effect of their changes on analyte ion concentrations. In the first set of simulations, for which negative analyte ion formation was assumed to occur via electron capture, $S_{e P}$ was varied over the same range $\left(10^{14}-10^{17} \mathrm{~s}^{-1}\right)$ as in the previous study of charged particle number densities. Despite the approximate $40 \times$ increase in $\left[e_{\text {therm }}^{-}\right]$and $\left[P^{+}\right]$(see Figure 3), Figure 5 indicates that $\left[A^{-}\right]$and $\left[A^{+}\right]$each changes by only about a factor of 2 . This can be rationalized by considering the earlier result-analyte ion number densities are effectively determined by the ratio of their ion formation and recombination rates. Consequently, there is little variation in $\left[A^{-}\right]$and $\left[A^{+}\right]$; any change in ion formation rate due to an alteration in $\left[e_{\text {therm }}^{-}\right]$or $\left[P^{+}\right]$is cancelled by a corresponding change in recombination rate since their number densities remain essentially equal over the range of $S_{e P}$ according to Figure 3 .

The effects of changes in source flow rate and volume were examined in the second set of simulations; the electron-ion pair generation rate $S_{e P}$ was fixed at a nominal value of $10^{15} \mathrm{~s}^{-1}$ near the optimum response as indicated in Figure 5. In the first case, the source volume was reduced by a factor of 4 by halving its diameter; however, $\Lambda$ was only reduced from $\sim 0.30$ to $\sim 0.26$ because optimum discharge performance requires the spacing between end plates to remain fixed. In the second case, the source pumping has been

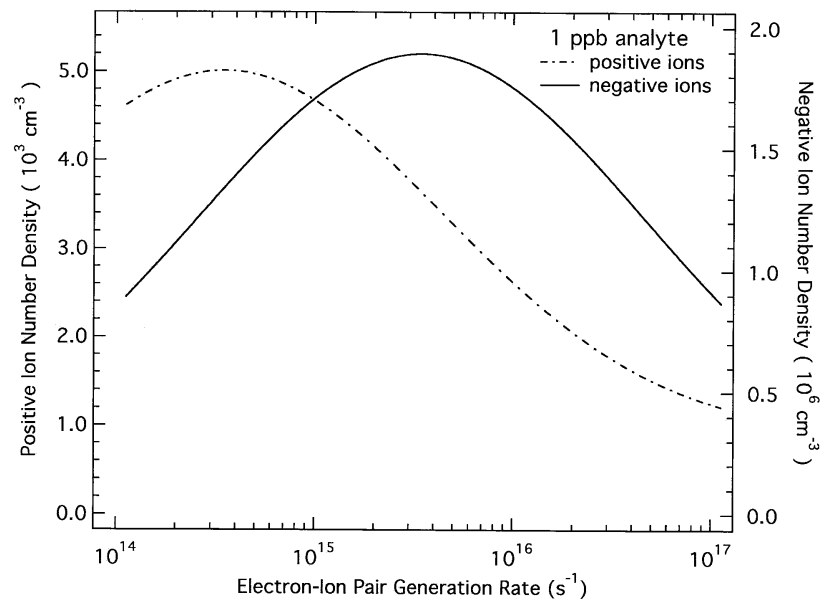

Figure 5. Variation of $\left[A^{-}\right]$and $\left[A^{+}\right]$with the electron-ion pair generation rate $S_{e P}$ for $\left[A_{0}\right]=1 \mathrm{ppb}$. increased to the extent that the flow rate is a factor of 2 greater than for standard conditions. To maintain the same $P_{A S G D I}$ as for standard conditions, the conductance of the inlet aperture $S C$ was also doubled. The results, which are presented in Table 3 as the analyte ion number density for the alternate to the nominal operating condition, $\left[A_{\text {alter }}^{ \pm}\right] /\left[A_{\text {nom }}^{ \pm}\right]$, indicate that the ratio increases in both cases. Examination of numerical output from the simulations reveals that the beneficial effect is due to the increase in neutral analyte number density in the source: in the first case, the source volume is reduced while the analyte inlet rate remains fixed, whereas in the second case the volume is constant but more analyte is introduced per unit time. Although increasing $F$ and reducing $V$ each produces a higher ventilation rate (with the latter also yielding a larger diffusion rate since $\Lambda$ is reduced), their effect on ion loss rate is minimal since the recombination rate still remains significantly larger.

Dynamic range and matrix effects. Although the efficiency of the ASGDI source is largely independent of the number densities for reagent species (i.e., $e_{\text {therm }}^{-} P^{+}$, $R^{-}$), the upper limit of its linear dynamic range $\left(\mathrm{LDR}_{\text {Ulim }}\right)$ is directly related to those quantities. When $\left[A_{0}\right]=1 \mathrm{ppb}$, simulation indicates that there is negligible depletion of the electron and reagent ion number densities as compared with quiescent discharge conditions, suggesting that $\mathrm{LDR}_{\text {Ulim }}$ is at least that large. $\mathrm{LDR}_{\mathrm{ULim}}^{\mathrm{eC}}$ can be estimated by rewriting eq 15 to reflect the relatively constant negative charge density and using the approximation $\left[e_{\text {therm }}^{-}\right] \approx\left[P^{+}\right]$to yield (for negative ions formed via electron capture):

$$
\begin{aligned}
\frac{\left[A^{-}\right]}{[A]} & =\frac{k_{e C}\left(\left[e_{\text {therm }}^{-}\right]-\left[A^{-}\right]\right)}{R_{\text {pos-neg }}\left[P^{+}\right]} \\
& \approx \frac{k_{e C}\left(\left[e_{\text {therm }}^{-}\right]-\left[A^{-}\right]\right)}{R_{\text {pos-neg }}\left[e_{\text {therm }}^{-}\right]} \\
& =\frac{k_{e C}}{R_{\text {pos-neg }}}\left(\frac{1}{1+\frac{k_{e C}[A]}{R_{\text {pos-neg }}\left[e_{\text {therm }}^{-}\right]}}\right)
\end{aligned}
$$

Eq 26 can then be rearranged and expanded to give

$$
\begin{aligned}
{\left[A^{-}\right]=[A] \frac{k_{e C}}{R_{\text {pos-neg }}}\left(1-\frac{k_{e C}[A]}{R_{\text {pos-neg }}\left[e_{\text {therm }}^{-}\right]}\right.} \\
\left.+\left[\frac{k_{e C}[A]}{R_{\text {pos-neg }}\left[e_{\text {therm }}^{-}\right]}\right]^{2}-\ldots\right)
\end{aligned}
$$


Table 3. Effect of alternate operating parameters on analyte ion concentrations ${ }^{\mathrm{a}}$

\begin{tabular}{llcc}
\hline & & {$\left[\mathrm{A}_{\text {alter }}^{-}\right] /\left[\mathrm{A}_{\text {nom }}^{-}\right]$} & {$\left[\mathrm{A}_{\text {alter }}^{+}\right] /\left[\mathrm{A}_{\text {nom }}^{+}\right]$} \\
\hline \hline$F_{\text {std }}$ & $V_{\text {std }} / 4$ & 1.93 & 1.91 \\
$F_{\text {std }} \times 2$ & $V_{\text {std }}$ & 1.43 & 1.63 \\
\hline
\end{tabular}

${ }^{\mathrm{a}} S_{e P}=10^{15} \mathrm{~s}^{-} 1 ; A^{-}$via electron capture, $k_{e C}=10^{-7} \mathrm{~cm}^{3}-\mathrm{s}^{-1} ; A^{+}$via ion-molecule reaction, $k_{i M}=10^{-9} \mathrm{~cm}^{3}-\mathrm{s}^{-1}$

A $1 \%$ deviation from linearity in $\left[A^{-}\right]$(formed via electron capture) at the upper end of the analyte dynamic range would then be predicted to occur when

$$
\frac{k_{e C}[A]}{R_{\text {pos-neg }}\left[e_{\text {therm }}^{-}\right]}=0.01
$$

Using the values obtained previously gives $[A]=3.7 \times$ $10^{8} \mathrm{~cm}^{-3}$; dividing $[A]$ by eq 20 yields the analyte concentration external to the source corresponding to $\mathrm{LDR}_{\mathrm{ULim}}^{\mathrm{eC}}$ :

$$
\begin{aligned}
\mathrm{LDR}_{\mathrm{ULim}}^{\mathrm{eC}} & =\frac{[A]}{[A] /\left[A_{0}\right]}=\frac{3.7 \times 10^{8} \mathrm{~cm}^{-3}}{6.3 \times 10^{-5}} \\
& =\left[A_{0}\right]=5.9 \times 10^{12} \mathrm{~cm}^{-3}=2.4 \times 10^{2} \mathrm{ppb}
\end{aligned}
$$

Previous experimental results for TNT showed a linear dynamic range from $\sim 1$ pptr to $100 \mathrm{ppb}$ [39]; the actual $\mathrm{LDR}_{\mathrm{ULim}}^{\mathrm{eC}}$ was not determined. A similar analysis for positive ions produced via ion-molecule reaction gives LDR ULim:

$$
\begin{aligned}
\mathrm{LDR}_{\mathrm{ULim}}^{\mathrm{iM}} & =\frac{[A]}{[A] /\left[A_{0}\right]}=\frac{6.0 \times 10^{11} \mathrm{~cm}^{-3}}{6.3 \times 10^{-5}} \\
& =\left[\mathrm{A}_{0}\right]=9.5 \times 10^{15} \mathrm{~cm}^{-3}=3.9 \times 10^{2} \mathrm{ppm}
\end{aligned}
$$

The higher $\mathrm{LDR}_{\text {Ulim }}$ for positive ions reflects the fact that the assumed ion-molecule reaction rate is slower than the electron capture rate; however, the overall linear dynamic ranges are comparable due to the superior sensitivity of negative ions formed via the latter process.

As noted earlier, loss of analyte ions can result from their ion-molecule reactions in the source with neutral matrix species $M$. The number density of matrix species at which such effects will alter $\left[A^{-}\right] /\left[A_{0}\right]$ can be estimated by again simplifying eq 8 , but with $\left[M_{0}\right] \neq 0$, to give:

$$
\frac{\left[A^{-}\right]}{[A]}=\frac{\frac{S C}{V}}{R_{\text {pos-neg }}\left[P^{+}\right]+k_{i M}[M]}
$$

For example, a $1 \%$ change in $\left[A^{-}\right] /[A]$ is predicted to occur when

$$
[M]=0.01 \cdot \frac{R_{\text {pos-neg }}\left[P^{+}\right]}{k_{i M}}
$$

Using previous values for the requisite parameters gives $[M]=4.6 \times 10^{10} \mathrm{~cm}^{-3}$. Simplifying eq 7 using the assumptions $D_{n} / \Lambda^{2}, F / V \gg k_{i M}\left[A^{+}\right], k_{i M}\left[A^{-}\right]$gives the steady-state expression for $[M] /\left[M_{0}\right]$,

$$
\frac{[M]}{\left[M_{0}\right]} \approx \frac{\frac{S C}{V}}{\frac{D_{n}}{\Lambda^{2}}+\frac{F}{V}}
$$

Dividing $[M]$ by eq 29 then yields the matrix concentration external to the source, $\left[M_{0}\right]=4.9 \mathrm{ppm}$. Similar analysis for $\left[A^{+}\right] /\left[A_{0}\right]$ via eq 9 gives $\left[M_{0}\right]=63 \mathrm{ppm}$.

\section{Conclusions}

As with conventional chemical ionization sources, ionization in the ASGDI source occurs primarily via electron- and ion-molecule reactions. In contrast to standard CI and corona APCI sources, however, essentially equal concentrations for charged particles of both polarities are present in the reduced pressure atmospheric sampling glow discharge. Furthermore, the ion and electron number densities are sufficiently high $\left(\sim 10^{10}\right.$ $\mathrm{cm}^{-3}$ ) that ion losses via drift and diffusion are negligible compared with ion-ion or ion-electron recombination. Therefore, analyte ion concentrations are not described by simple pseudo first-order kinetic expressions as employed for treating conventional $\mathrm{CI}$ or by similar expressions coupled with unipolar charge drift as used for modeling APCI. Consequently, the overall ASGDI dynamics are modeled by combining a system of coupled differential equations describing the rates of quiescent source processes and formation/loss for analyte species with the physical parameters of the source. Steady-state approximations of the equations indicate that, as in other plasma ionization sources, the ionization efficiency is essentially determined by the ratio of the relevant ion formation and recombination rates. Furthermore, numerical modeling of the charged particle dynamics indicates that the secondary electron thermalization rate is sufficiently greater than the overall electron loss rate to yield a high thermal/secondary electron ratio at steady-state. Therefore, the efficiency for negative analyte ion formation via electron capture is generally superior to that for positive analyte ion generation via ion-molecule reaction since the electron capture/ion-molecule reaction rate coefficient ratio is normally quite large and the ion-electron recombination rate coefficient is about an order of magnitude greater than that for ion-ion recombination. However, the efficiency for positive analyte ion formation should be equal to or better than that for negative analyte ions when both ionization processes occur via ion-molecule 
reaction processes (with comparable rate coefficients), since the negative reagent ion density is considerably less than that for positive reagent ions. A further consequence of the ion formation/recombination rate dependent ionization efficiency is that, for a given analyte concentration, there is relatively little variation in analyte ion number density with initial electron-ion pair formation rate in the discharge. That is, any change in ion formation rate due to an alteration in thermal electron or reagent ion concentration is cancelled by a corresponding change in recombination rate since their number densities remain essentially equal. However, simulation results also suggest that analyte ion number densities might be enhanced by increasing the ventilation rate or reducing the radius of the source. The beneficial effect is due to the increase in neutral analyte number density in the source: in the first case, the volume is constant but more analyte is introduced per unit time, whereas in the second case, the source volume is reduced while the analyte inlet rate remains fixed.

\section{Acknowledgments}

The research presented here was supported by the Division of Chemical Sciences, Geosciences, and Biosciences, Office of Basic Energy Sciences, U.S. Department of Energy at Oak Ridge National Laboratory, managed and operated by UT-Battelle, LLC under contract DE-AC05-00OR22725. The authors greatly appreciate the valuable comments of S. A. McLuckey, G. J. Van Berkel, K. G. Asano, and G. P. Jackson in the preparation and review of the manuscript.

\section{References}

1. Carroll, D. I.; Dzidic, I.; Stillwel, R. N.; Horning, M. G.; Horning, E. C. Subpicogram Detection System for Gas-Phase Analysis Based Upon Atmospheric-Pressure Ionization (API) Mass-Spectrometry. Anal. Chem. 1974, 46, 706-710.

2. Ketkar, S. N.; Dulak, J. G.; Fite, W. L.; Buchner, J. D.; Dheandhanoo, S. Atmospheric-Pressure Ionization Tandem MassSpectrometric System for Real-Time Detection of Low-Level Pollutants in Air. Anal. Chem. 1989, 61, 260-264.

3. McLuckey, S. A.; Goeringer, D. E.; Asano, K. G.; Vaidyanathan, G.; Stephenson, J. L., Jr. High Explosives Vapor Detection by Glow Discharge-Ion Trap Mass Spectrometry. Rapid Commun. Mass Spectrom 1996, 10, 287-298.

4. Thomson, B. A.; Davidson, W. R.; Lovett, A. M. Applications of a Versatile Technique for Trace Analysis-AtmosphericPressure Negative Chemical Ionization. Environ. Health Perspect. 1980, 36, 77-84.

5. Evans, J. E.; Arnold, J. T. How to Monitor Organics in Air. Environ. Sci. Technol. 1975, 9, 1134-1138.

6. Wise, M. B.; Guerin, M. R. Direct Sampling MS for Environmental Screening. Anal. Chem. 1997, 69, A26-A32.

7. Weickhardt, C.; Boesl, U.; Schlag, E. W. Laser Mass-Spectrometry for Time-Resolved Multicomponent Analysis of Exhaust-Gas. Anal. Chem. 1994, 66, 1062-1069.

8. Boesl, U.; Weinkauf, R.; Weickhrdt, C.; Schlag, E. W. Laser Ion Sources for Time-of-Flight Mass-Spectrometry. Int. J. Mass Spectrom. Ion Processes 1994, 131, 87-124.

9. Franzen, J.; Frey, R.; Nagel, H. Fast Monitoring of Motor Exhaust Components by Resonant Multiphoton Ionization and Time-of-Flight Mass-Spectrometry. J. Mol. Struct. 1995, 347, 143-151.

10. Butcher, D. J.; Goeringer, D. E.; Hurst, G. B. Real-Time Determination of Aromatics in Automobile Exhaust by Single Photon Ionization Ion Trap Mass Spectrometry. Anal. Chem. 1999, 71, 489-496.

11. Carroll, D. I.; Dzidic, I.; Horning, E. C.; Stillwell, R. N. Atmospheric-Pressure Ionization Mass-Spectrometry. Appl. Spectrosc. Rev. 1981, 17, 337-406.

12. Dzidic, I.; Carroll, D. I.; Stillwell, R. N.; Horning, E. C. Comparison of Positive-Ions Formed in Nickel-63 and Corona Discharge Ion Sources Using Nitrogen, Argon, Isobutane, Ammonia, and Nitric-Oxide as Reagents in AtmosphericPressure Ionization Mass-Spectrometry. Anal. Chem. 1976, 48, 1763-1768.

13. Horning, E. C.; Horning, M. G.; Carroll, D. I.; Dzidic, I.; Stillwell, R. N. New Picogram Detection System Based on a Mass-Spectrometer with an External Ionization Source at Atmospheric-Pressure. Anal. Chem. 1973, 45, 936-943.

14. Siegel, M. W.; Fite, W. L. Terminal Ions in Weak AtmosphericPressure Plasmas-Applications of Atmospheric-Pressure Ionization to Trace Impurity Analysis in Gases. J. Phys. Chem. 1976, 80, 2871-2881.

15. McLuckey, S. A.; Glish, G. L.; Asano, K. G.; Grant, B. C. Atmospheric Sampling Glow-Discharge Ionization Source for the Determination of Trace Organic-Compounds in Ambient Air. Anal. Chem. 1988, 60, 2220-2227.

16. Chambers, D. M.; Mcluckey, S. A.; Glish, G. L. Role of Gas-Dynamics in Negative-Ion Formation in an Atmospheric Sampling Glow-Discharge Ionization Source. Anal. Chem. 1993, 65, 778-783.

17. Kambara, H. Sample Introduction System for AtmosphericPressure Ionization Mass-Spectrometry of Non-Volatile Compounds. Anal. Chem. 1982, 54, 143-146.

18. Dougherty, R. C.; Dalton, J.; Biros, F. J. Negative Chemical Ionization Mass-Spectra of Polycyclic Chlorinated Insecticides. Org. Mass Spectrom. 1972, 6, 1171.

19. Hunt, D. F.; Crow, F. W. Electron-Capture Negative-Ion Chemical Ionization Mass-Spectrometry. Anal. Chem. 1978, 50, 1781-1784.

20. Knighton, W. B.; Sears, L. J.; Grimsrud, E. P. High-Pressure Electron Capture Mass Spectrometry. Mass Spectrom. Rev. 1995, 14, 327-343.

21. Sears, L. J.; Campbell, J. A.; Grimsrud, E. P. Ionization Dynamics within the High-Pressure Electron-Capture MassSpectrometer-the Unusual Spectra of Derivatized Polycyclic Aromatic-Amines and Perchlorinated Unsaturated-Hydrocarbons. Biomed. Environ. Mass Spectrom. 1987, 14, 401-415.

22. Siegel, M. W.; Mckeown, M. C. Ions and Electrons in ElectronCapture Detector-Quantitative Identification by Atmospheric-Pressure Ionization Mass-Spectrometry. J. Chromatogr. 1976, 122, 397-413.

23. Busman, M.; Sunner, J.; Vogel, C. R. Space-Charge-Dominated Mass-Spectrometry Ion Sources-Modeling and Sensitivity. J. Am. Soc. Mass Spectrom. 1991, 2, 1-10.

24. Good, A.; Durden, D. A.; Kebarle, P. Mechanism and Rate Constants of Ion-Molecule Rfactions Leading to Formation of $\mathrm{H}+(\mathrm{H} 2 \mathrm{o}) \mathrm{N}$ in Moist Oxygen and Air. J. Chem. Phys. 1970, 52, 222.

25. Chapman, B. Glow Discharge Processes; Wiley: New York, 1980, pp. 32-55.

26. Oster, T.; Kuhn, A.; Illenberger, E. Gas-Phase Negative-Ion Chemistry. Int. J. Mass Spectrom. Ion Processes 1989, 89, 1-72.

27. Marion, J. B.; Gray, D. E. American Institute of Physics Handbook; 3rd ed; McGraw-Hill: New York, 1972, p 184.

28. Warman, J. M.; Sauer, M. C. Investigation of Electron Thermalization in Irradiated Gases Using Ccl4 as an ElectronEnergy Probe. J. Chem. Phys. 1975, 62, 1971-1981. 
29. Pack, J. L.; Phelps, A. V. Electron Attachment and Detachment .2. Mixtures of $\mathrm{O}_{2}$ and $\mathrm{CO}_{2}$ and of $\mathrm{O}_{2}$ and $\mathrm{H}_{2} \mathrm{O}$. J. Chem. Phys. 1966, 45, 4316.

30. Phelps, A. V. Laboratory Studies of Electron Attachment and Detachment Processes of Aeronomic Interest. Canad. J. Chem. 1969, 47, 1783.

31. O'Hanlon, J. F. A User's Guide to Vacuum Technology; John Wiley and Sons: New York, 1980, pp 37-43.

32. McDaniel, E. W. Collision Phenomena in Ionized Gases; Wiley: New York, 1964, pp 498-506.

33. Mason, E. A.; McDaniel, E. W. Transport Properties of Ions in Gases; Wiley-Interscience: New York, 1988, p 245.

34. Mitchell, J. B. A.; McGowan, J. W. In Physics of Ion-Ion and Electron-Ion Collisions; Brouilliard, F.; McGowan, J. W., Eds.; Plenum Press: New York, 1983, pp 296-297.
35. Smith, D.; Adams, N. G. In Physics of Ion-Ion and Electron-Ion Collisions; Brouilliard, F.; McGowan, J. W., Eds.; Plenum Press: New York, 1983, pp 501-529.

36. Klots, C. E. Rate Constants for Unimolecular Decomposition at Threshold. Chem. Phys. Lett. 1976, 38, 61-64.

37. Harrison, A. G. Chemical Ionization Mass Spectrometry; CRC Press: Boca Raton, FL, 1983.

38. Sunner, J.; Nicol, G.; Kebarle, P. Factors Determining Relative Sensitivity of Analytes in Positive Mode AtmosphericPressure Ionization Mass-Spectrometry. Anal. Chem. 1988, 60, 1300-1307.

39. Grant, B. C.; Asano, K. G.; Glish, G. L.; McLuckey, S. A. Proceedings of the 36th Annual Conference on Mass Spectrometry and Allied Topics, San Francisco, CA, June 1988; p 47. 